\title{
Recurrent Female Reproductive System
} Carcinoma

National Cancer Institute

\section{Source}

National Cancer Institute. Recurrent Female Reproductive System Carcinoma. NCI

Thesaurus. Code C7620.

The reemergence of female reproductive system carcinoma after a period of remission. 